\title{
DEVELOPMENT OF THE MUCUS-SECRETING ELEMENTS IN HUMAN LUNG
}

\author{
BY \\ U. BUCHER* AND LYNNE REID \\ From the Institute of Diseases of the Chest and Brompton Hospital, London
}

(RECEIVED FOR PUBLICATION JULY 11, 1961)

Recent interest in chronic bronchitis has necessarily drawn attention to the functional and anatomical aspects of mucus secretion. In the bronchial tree mucus is secreted by goblet cells in the surface epithelium (sometimes called "intraepithelial glands") and by the tubulo-acinar glands external to the muscle layer and opening to the bronchial surface by an excretory duct. The latter are herein referred to as glands and the former as goblet cells.

This paper describes the intrapulmonary development of the glands and the goblet cells in the foetal human lung and their number and distribution, which last is expressed by reference to the number of bronchial generations and in relation to the extension of cartilage at a given age. Previous papers have described $(a)$ the pattern of branching of the intrasegmental bronchial tree and cartilage development (Bucher and Reid, 1961), and (b) the mucous glands in the trachea (Thurlbeck, Benjamin, and Reid, 1961).

\section{HISTORICAL}

Mucous glands first appear in the fourth month of foetal life as small solid buds emerging from the basal cells of the bronchial epithelium and penetrating into the subepithelial layers (Diezel, 1938 ; Brites, 1929). They are usually formed at the bottom or in the lateral wall of epithelial creases (Brenek, 1941), and develop a mixed tubulo-acinar form (Arey, 1954 ; Engel, 1947). In a foetus of $500 \mathrm{~mm}$. crown-rump length (11 weeks), Brenek observed glands in both lungs, 10 anlagen on the left but only four on the right. These buds, about 15 to $50 \mu$ in diameter, were bulging the basal membrane and extended to the level at which hyalinization of the ground substance of the cartilage ended. While Engel (1947) also reports almost complete coincidence between cartilage and glands in children, Brouet and

*Medizinische Universitätsklinik, Berne, Switzerland. The work reported in this paper was carried out during tenure of a grant from the Janggen-Poehn Foundation, St. Gallen. Reference was made to it in a paper delivered to the Thoracic Society at made to it in
Edinburgh, 1960.
Chretien (1956) found that the glands did not extend as far distally as did cartilage.

Probably at birth the glands do not yet possess their final shape (Diezel, 1938; Engel, 1947: Leonardelli, Pignataro, and Vago, 1957; Gammarrota, 1954). From the literature it is not clear whether post-natal development chiefly consists in an increase in the number of glands or in the size of individual glands.

Brites (1929) reported that goblet cells are first detectable in the tracheal epithelium of foetuses of 5 months, but Thurlbeck et al. (1961) found that intracellular vacuoles were recognizable in a 12-week foetus and that at $13 \frac{1}{2}$ weeks welldeveloped goblet cells containing P.A.S.-positive material could be identified.

\section{MATERIALS AND METHODS}

The present investigations have been carried out with the same material (Table I, p. 221) as the previously mentioned study on bronchial branching and cartilage development (Bucher and Reid, 1961). The lungs had been cut in serial sections of $7 \mu$ thickness. In the parts specially selected for investigation of the glands every section, occasionally only every third, was available, giving a maximum distance between sections of $21 \mu$. For the analysis of the morphological and histochemical behaviour of the glands at various ages, alternate sections were stained with haematoxylin-eosin and by the periodic-acid-Schiff (P.A.S.) method. In some foetuses further staining included alcian blue, iron haematoxylin, and toluidine blue.

The study of gland and goblet cell distribution entailed (1) tracing the ultimate or most distal gland bud in selected bronchi, usually axial pathways, i.e., the longest possible (Hayward and Reid, 1952), in foetuses of different ages, and relating this level to that of cartilage extension ; (2) counting of glands and glandular buds in selected parts of several pathways, in order to assess gland density.

For this purpose selected bronchi were sectioned at right angles to their long axes; every section was examined, and all the glands within a generation, i.e., any length of bronchial tree between consecutive branches, were counted. Similar estimates were made for the goblet cells. 


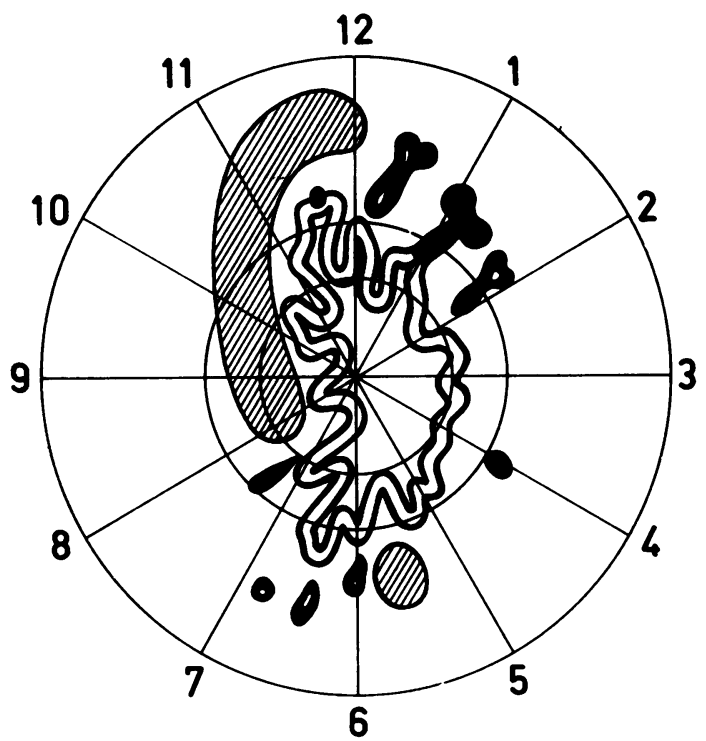

FIG. 1.-Diagrammatic representation of cross-section of bronchus (ducts and glands black, cartilage shaded). The circumference is considered to be divided into 12 segments as on a clock face. These structures within any segment are then translated to lined paper as in Fig. 2.

In examining the cross-section of the bronchus to establish the density of glands, it was divided into 12 roughly equal segments as on a clock dial (Fig. 1) and the position of each gland in relation to these segments noted on a diagram as in Fig. 2. The sites at which ducts open on to the epithelial surface are marked with an arrow (Fig. 2). In older foetuses, where the glands are contiguous and therefore more difficult to distinguish, it was necessary to rely on the ducts for estimating the number

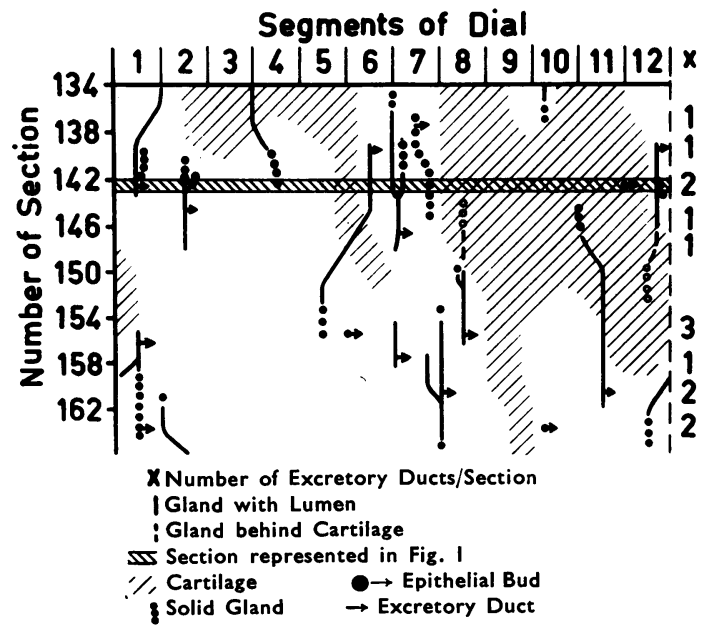

FIG. 2.-The section shown in Fig. 1 is represented on the lined paper by the shaded strip passing horizontally across the diagram at level 142 in the left-hand column. of glands. The position of the cartilage also was marked on the plan (Fig. 2). From such a plan drawn for any given generation the following can be established: The total number of glands; the general size, form, and position of the glands; the position of the ducts in relation to the glandular body; the concentration of glands in relation to points of bronchial branching; and the topographical relation of the glands to the cartilage.

From the total number of glands for the distance over which they have been counted (number of sections $\times 7 \mu$ ) and from an estimate of the mean radius of the bronchus, their density (glands $/ \mathrm{mm}^{2}$ ) can be calculated. The formula is

$$
\mathrm{x}=\frac{\mathrm{n}}{\mathrm{L} \times 2 \pi \times \sqrt{\frac{\mathrm{a}^{2}+\mathrm{b}^{2}}{2}}}
$$

where $x=$ density (glands/sq. $\mathrm{mm}$.)

$\mathrm{n}=$ total number in a given generation

$\mathrm{L}=$ length of this generation (in $\mathrm{mm}$.)

$\mathrm{a}=$ longest radius of bronchus (the average of various levels - the diameter being measured from the basement membrane) in $\mathrm{mm}$.

$\mathrm{b}=$ shortest radius (average as above) in $\mathrm{mm}$. Note that $\frac{\sqrt{a^{2}+b^{2}}}{2}$ gives a mean radius.

\section{RESULTS}

Development of an Individual Gland

Recognition of Gland.-The first suggestion of the formation of a gland is an accumulation of cells with dark nuclei forming a small, sharply defined cluster within the epithelium just internal to the basement membrane (Fig. 3a), the bronchial epithelium at this stage often consisting of only one layer of cells. The basement membrane is bulged by the growing cluster which gradually forms a solid bud penetrating into the subepithelial layers. This early solid stage is not often observed and seems to be of short duration.

Soon after a bud is formed a lumen can be detected giving rise to a primitive tubular gland (Fig. 3b). Once a gland is well developed the duct opens on to the surface at the bottom of a crypt which, as it is not seen in the preglandular cluster or at the solid bud stage, possibly represents dilatation of the superficial part of the duct. After penetrating the muscle layer the duct passes from a radial into a more tangential or longitudinal direction, particularly in the case of glands lying internal to cartilage. The growth may be in a proximal or distal direction. Glands developing between cartilage very often follow its edge and even when in a simple tubular stage extend behind it. This can already be observed in a foetus of only 14 weeks. Dichotomous division or lateral budding ultimately gives way to the formation of an acinar pattern. 


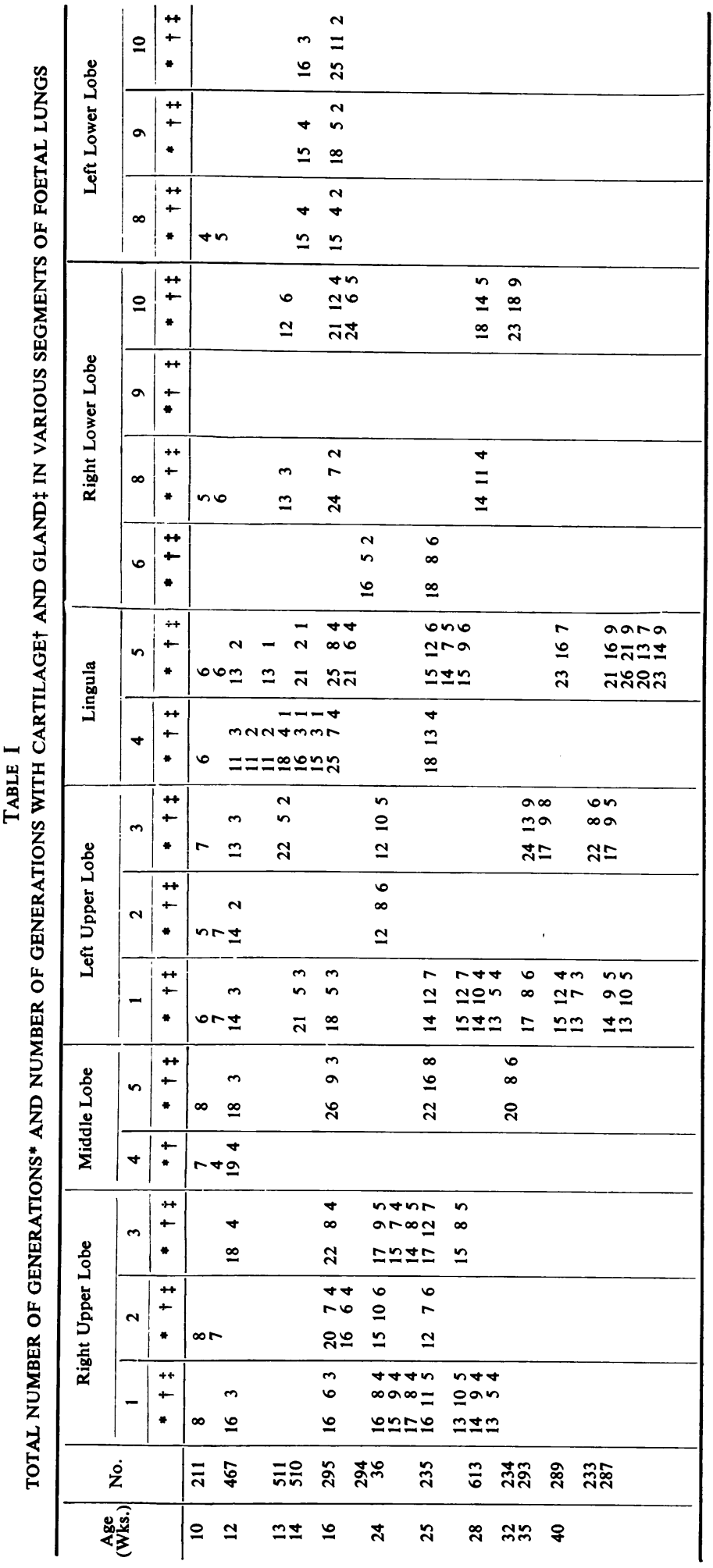



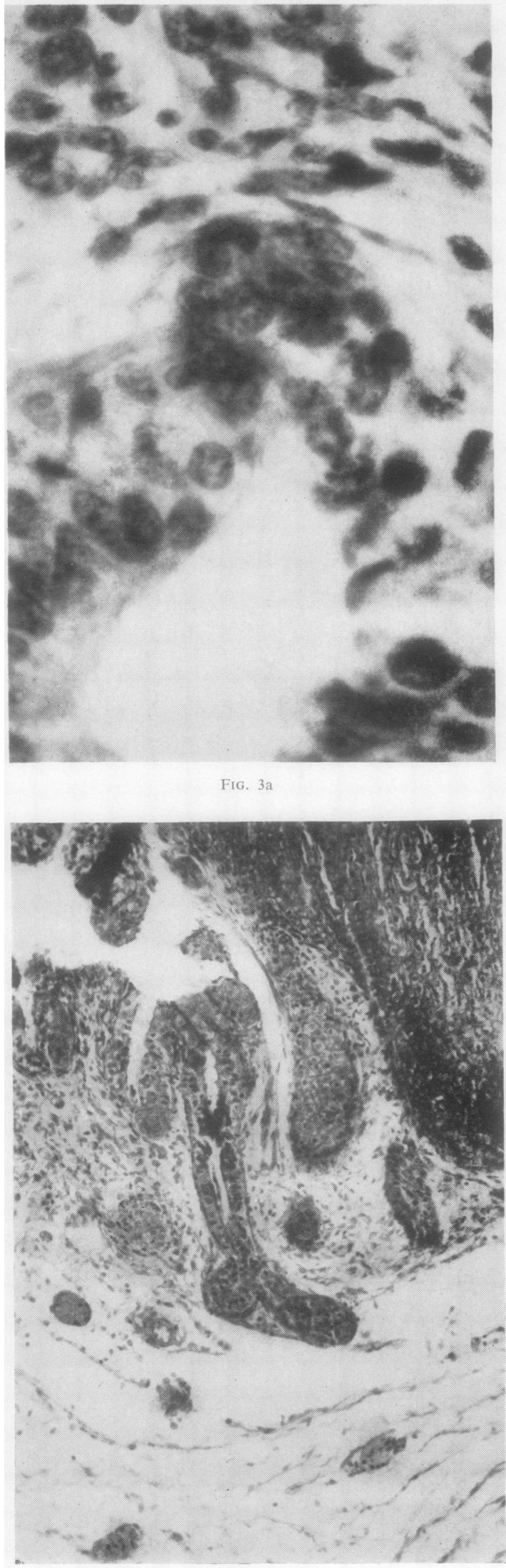

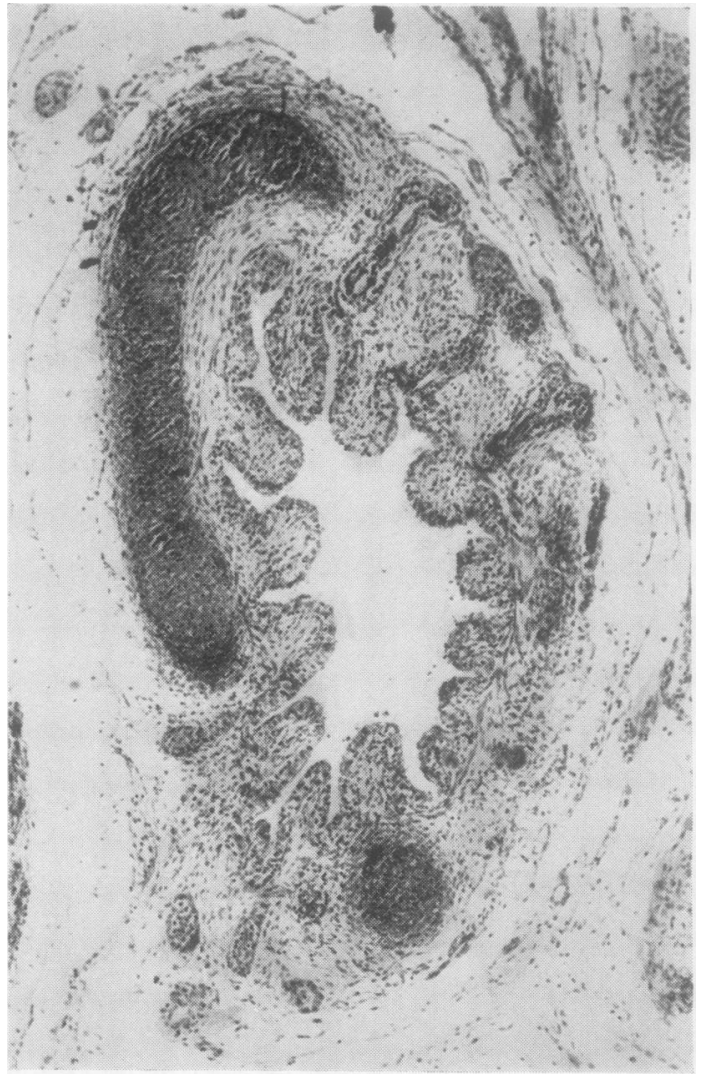

FIG. 3c

FIG. 3.- (a) Solid bud of epithelium from which branching duct (i) develops which may soon lie external to cartilage (c). $(a, \times 80 ; b$ and $c, \times 30$.)

Histochemical Features. - The cells of the early gland mass are polygonal with a round central nucleus and slightly granular cytoplasm; they retain this appearance even after the lumen is formed. Mitoses are not frequent. Special stains reveal mucinous substances in the cells of the glands at this early stage; the first small buds do not show any P.A.S.-positive material, but as soon as a lumen appeared it could be detected in the form of fine granules, dark purple in colour, between nucleus and cell surface. Before this a few cells contain granules which give a bright reaction with alcian blue or mucicarmine but not with P.A.S.

As the glands branch and develop acini, some cells are distended with mucus while others contain granules which stain for mucus, others again being quite free. With haematoxylin-eosin stain, the cytoplasm of the distended cells appears foamy and the collection of mucus may be so large as to flatten the nucleus. Such cells are less frequent the less developed the glands, but after 24 weeks they may form acini in proximal glands, although distal ones contain only granules. 


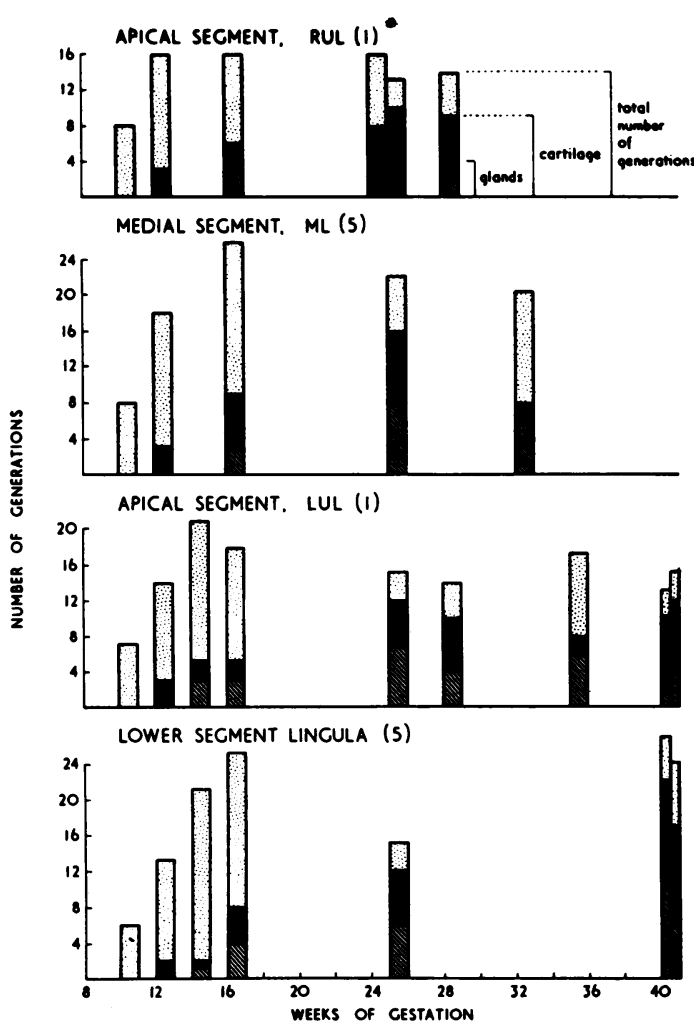

- Numbers refer to segments as described in Thorox (1950). S. 222

FIG. 4.-Relation of gland extent to that of cartilage and number of generations.

As the glands become tubular the epithelium close to the bronchial surface consists of cylindrical cells with oval nuclei which ultimately resemble those of the surface epithelium, i.e., ciliated columnar cells, except that goblet cells are rare on the surface and numerous in the duct. The crypt or dilated part of the duct may sometimes penetrate deeply through the muscle layer. Further from the bronchial lumen the cells lining the duct are more cuboidal, have a central nucleus, and may be arranged in a layer one or two cells deep, and cilia can no longer be detected.

\section{Distribution of Glands in the Bronchial TREE}

Relation to the Age of Foetus.-In foetuses of 10 and 12 weeks no gland buds could be detected in trachea or bronchi, the first being found at 13 weeks, when both solid buds and primitive tubular glands were seen. At the end of the tubules accumulations of small dark cells could sometimes be seen, and this was probably the region of most growth.

At 14 weeks the number of tubular glands had increased, many of them showing lateral buds which also included a lumen, usually delineated by a fringe of P.A.S.-positive material.

The mucus-staining cells increase over the next three weeks. By the twenty-fourth and twentyfifth week glands in the main bronchi had typ:cal acinar structure, some consisting exclusively of mucus-staining cells. The glands in more distal bronchi are more primitive in their structure, being still simple tubules.

Solid buds or intra-epithelial preglandular collections of cells are rather rare at this age, although with serial sections one could occasionally be detected even in a 28-week foetus. But if at this age the distal glands are still simple branched tubules, the more proximal glands show a complex acinar structure resembling the adult type though in miniature.

Relation to Cartilage.-Fig. 4 and Table I show the relation of the ultimate gland or gland bud to the last piece of cartilage and to the number of intrasegmental branches of the bronchial tree. The method for counting generations is described on page 208. Glands extend along the bronchial tree in a similar way to cartilage up to the twenty-fourth week, after which no significant extension is observed.

In the shorter pathways, e.g., in the apical segments of both upper lobes, the average number of generations containing glands is between four and six for different lungs (the number of intrasegmental generations is 13 to 16 , including eight

TABLE II

DENSITY OF GLANDS AT VARIOUS FOETAL AGES

\begin{tabular}{|c|c|c|c|c|c|c|c|}
\hline Age in Weeks & $\begin{array}{c}\text { Second } \\
\text { Presegmental } \\
\text { Bronchus }\end{array}$ & $\begin{array}{c}\text { Presegmental } \\
\text { Bronchus }\end{array}$ & $\begin{array}{l}\text { Segmental } \\
\text { Bronchus }\end{array}$ & $\begin{array}{l}\text { Second } \\
\text { Order }\end{array}$ & $\begin{array}{l}\text { Third } \\
\text { Order }\end{array}$ & $\begin{array}{l}\text { Fourth } \\
\text { Order }\end{array}$ & Pathways \\
\hline $\begin{array}{l}13 \\
14 \\
16 \\
16 \\
28 \\
35 \\
40 \\
40\end{array}$ & $25 *(15)$ & $\begin{array}{ll}58 & (129) \\
15 \cdot 3 & (21)\end{array}$ & $\begin{array}{lr}13 & (3) \\
51 & (34) \\
34 & (58) \\
43 \cdot 2 & (150) \\
21 & (71)\end{array}$ & \begin{tabular}{cl}
$(1$ & \multicolumn{2}{c}{ single gl. } \\
$=$ & last gl.) \\
37 & $(35)$ \\
18 & $(12)$ \\
& \\
22 & $(69)$ \\
17 & $(90)$
\end{tabular} & $\begin{array}{ll}15 & (74) \\
& \\
10 & (6) \\
18 & (8) \\
17 & (32)\end{array}$ & $\begin{array}{rr}10 & (41) \\
5 & (11)\end{array}$ & $\begin{array}{l}\text { Apical L.U.L. } \\
\text {," R.'’.L. } \\
\text { Lateral M.L.' } \\
\text { Apical L.U.L. } \\
\text { Superior lingula } \\
\text { Ap̈ical L.U.厃̈. }\end{array}$ \\
\hline
\end{tabular}

$*=$ No. of glands/sq. mm. No. in brackets $=$ absolute count. 
to 12 bronchi with cartilage). In longer pathways, e.g., in the middle lobe or lingula, the corresponding figures are six to nine generations with glands as compared with 16 to 20 with cartilage, in a total of 20 to 26 . Roughly speaking, a third of all the fully epithelialized generations along any pathway contain glands. There is much greater variation in cartilage than gland extension.

Density of Glands.-The number of glands within a bronchial generation depends on the age of a foetus, on the position of the generation, and on its length. Since length and diameter vary considerably, comparison must be made on the basis of density (glands per square millimetre of wall), which, although rough, gives a fair idea of the distribution (Table II).

Although at the age of 12 weeks no glands or buds are found in intrasegmental bronchi, the highest density of glands occurs at 14 weeks. In a segmental bronchus, the concentration was $50 / \mathrm{mm}^{2}$, dropping to 30 to 40 in the next generation. Taking the same pathways, the density at 28 weeks was about 40 in the first generation and 20 in the second, the diminution of 20 per mm. ${ }^{2}$ being due perhaps to an increase in the size of the lung. To investigate the change in area of the bronchial wall, the length of these bronchi at two ages was compared. Both length and circumference were considered, but during the period in question the increase in the latter was insignificant as compared with the former, and for this estimate it may be assumed that the increase in area of a generation is in direct proportion to the increase in its length. The maximum length of the apical segment of the left upper lobe, that is, of the whole of an axial pathway, is approximately doubled between 14 and 28 weeks, and the first generation at 28 weeks is more than four times as long as at 14 weeks; it would thus seem that growth is more pronounced in the more proximal generations of a segment. Similarly the surface area of the first generation is increased more than four times. Since the original density of glands $\left(51 / \mathrm{mm} .^{2}\right.$ area) must theoretically have been "diluted" or diminished to about $13 \mathrm{~mm} .{ }^{2}$, and whereas we found a real density of 43 glands $/ \mathrm{mm} .^{2}$ at 28 weeks, this suggested that new growth had occurred, for $30 / \mathrm{mm}$. of these glands or $75 \%$ must have been formed between 14 and 28 weeks. For the second generation a very similar figure $(68 \%)$ was calculated.

Although the number of generations investigated was small, the figures given in Table II indicate that the density of glands in the bronchial mucosa is highest at an early stage of development. At birth about 17 glands per $\mathrm{mm}^{2}$ were found in the third generation of two different segments.

Comparison of Number of Glands in Generations of a Bronchial Pathway.-As the figures in Table II show, gland density is highest in the most proximal generations of a bronchus; it decreases considerably from one generation to the next, though the difference is less pronounced in proximal generations than in the more distal ones.

Distribution of Glands in Single Generations.The distribution of glands along a single generation is uneven, being most concentrated in the neighbourhood of a division. In a single section no constant relationship to cartilage could be found, although cartilage tends also to be more concentrated near bifurcations.

Distribution of Goblet Cells.-Goblet cells, as well as glands, appear in the trachea and large bronchi at about 13 weeks. Between 12 and 24 weeks goblet cells are found only in the most proximal intrasegmental generations; they never spread as far as glands do. Only after 32 weeks could an occasional goblet cell be found in generations distal to the last gland. At term they sometimes reached the last level of cartilage, but no goblet cell was observed in a bronchiolus.

In general, then, goblet cells are very rare on the surface of foetal bronchi, although they are numerous in the crypts of the excretory ducts of the glands. Besides the typical goblet cells containing a large amount of mucus, ciliated cells are also found which contain a few small P.A.S.positive droplets just beneath the ciliated fringe, these being more frequently seen in later foetal life.

No difference in the number of goblet cells could be detected between stillborn foetuses and those which had breathed for a few hours or days.

\section{Observations on other Histological Features of THE Bronchial TREE}

Epithelium.--In the adult bronchial tree the epithelium is pseudostratified ciliated columnar. At 10 weeks' foetal age the tracheal epithelium is multilayered. A superficial layer of columnar cells can be distinguished, with a palely staining, somewhat granular, cytoplasm and round nuclei lying toward the superficial part of the epithelium, their tapering lower ends merging with the cells of the basal layer, which have a more irregular polygonal shape and contain numerous mitoses. Some of the superficial cells present a fringe of what may be adherent cilia. Between these two main layers a few spindle-shaped cells can be seen. 
At this stage some superficial cells include P.A.S.positive granules, although no drop-like inclusions comparable to the later goblet cells are seen. In the main bronchi the epithelium gradually diminishes in thickness to a single layer of cylindrical, and then more peripherally of cuboidal, cells. These cuboidal cells even cover the distal parts of the bronchial tree which at this stage are blind. By 13 weeks cilia can be detected even to the end of the bronchial tree.

By 24 weeks, following a pathway from the proximal generations towards the periphery, the ciliated epithelium with two or more layers of cells shows a gradual change to a single layer of columnar cells and then cuboidal cells still covered with cilia and containing centrally-placed round or oval-shaped nuclei. In the most peripheral parts the epithelial cells are flattened, free of cilia, and capillaries can be observed penetrating between these flattened cells and reaching the surface of the bronchiolar wall. By term ciliated cells reach the terminal bronchiolus.

The Basement Membrane. - The basement membrane can be recognized by a pale pink stain with the P.A.S. reaction at 10 weeks, but does not yet appear to be continuous, although by 12 weeks the basement membrane is well developed.

The appearance of the cartilage has been described separately (Bucher and Reid, 1961).

\section{COMMENT}

At birth a mean density of 17 glands per square millimetre was calculated for the third generation of two different segments. Since no exact data are available concerning gland density in the adult lung, only a crude calculation can be made of the changes which occur after birth. According to von Hayek (1953) there is one gland per square millimetre in a bronchus of medium size; our foetal estimate would suggest a lower figure for the adult, and accordingly it seems that new glands are formed after birth.

Engel (1947) and Brenek (1941) have postulated a relationship between the extent of the glands and of cartilage along the bronchial tree, but in the foetal lungs here investigated cartilage growth showed a high degree of variation, whereas the glands extended in fairly constant proportion to the number of generations, seemingly independent of the ultimate extension of cartilage.

\section{SUMMARY}

By the fourteenth week glands are evident, growing as solid buds from the basal layers of the surface epithelium; a short-lived phase as a lumen converts this to a tubule whose superficial part dilates to form a trough or crypt-like structure. The tubules branch and pass superficially to and behind the cartilage ; at their ends acini are well formed in the main bronchi by 24 weeks and include many cells distended with mucus. Some P.A.S.-positive material is seen as soon as a tubule is present.

The glands do not extend as far as cartilage along the bronchial tree, being found in roughly a third of all fully epithelialized generations along any pathway.

Gland density is greatest at 14 weeks, but, taking bronchial growth into account as well as gland density, it means that many new glands must form even in the large bronchi between, e.g., the fourteenth and twenty-eighth weeks, although many glands are already well differentiated. Gland density is highest in proximal bronchi and decreases toward the periphery, in a single generation being more concentrated at bifurcations. At term, for example, the concentration of glands in any of the first few intrasegmental bronchi may be between 10 and 20 per square millimetre; beyond this it falls off quite quickly. At the fourteenth week counts as high as 50 ducts per square millimetre may be found.

Goblet cells appear also at about the thirteenth week, but are easily seen only in proximal intrasegmental generations; only after the thirtysecond week may an occasional one be seen distal to the last gland. Even at term, none is found distal to the last plate of cartilage, i.e., in bronchioli, although some cells do contain fine granules which stain for mucus.

Cilia are seen in the trachea and main bronchi at 10 weeks, in most peripheral airways by 13 weeks.

Our thanks are due to Mr. A. Curd, of the Photographic Department of the Institute of Diseases of the Chest, Brompton Hospital, London, S.W.3, for the photomicrographs.

\section{REFERENCES}

Arey, L. B. (1954). Developmental Anatomy, 6th ed. Saunders, Philadelphia and London.

Brenek, I. (1941). Z. mikr.-anat. Forsch, 49, 525

Brites, G. (1929). C. R. Soc. Biol. (Paris), 102, 231.

Brouet, G., and Chretien, J. (1956). Bronches, 6, 7.

Bucher, U., and Reid, L. (1961). Thorax, 16, 207.

Diezel, W. (1938). Z. Menschl. Vererb.-u. Konstit.-Lehre, 21, 572.

Engel, S. (1947). The Child's Lung. Arnold London.

Engel, S. (1947). The Child's Lung. Arnold Lond

Gammarrota, V. (1954). Riv. Tuberc., 2, 125. . Sie menschliche Lunge. Springer, Berlin.

Hayward, J., and Reid, L. (1952). Thorax, 7, 89.

Leonardelli, G. B., Pignataro, O., and Vago, A. (1957). Bronches, 7, 580.

Thurlbeck, W. M., Benjamin, B., and Reid, L. (1961). Brit. J. Dis. Chest, 55, 49 and 54. 